\title{
BIFURCATION OF RESPONSE OF A NONLINEAR VISCOELASTIC SPHERICAL MEMBRANE
}

\author{
Alan Wineman \\ Department of Applied Mechanics and Engineering Science, The University of Michigan, Ann Arbor, \\ MI 48109, U.S.A.
}

(Received 2 March 1977; in revised form 26 July 1977; received for publication 27 September 1977)

\begin{abstract}
The quasistatic inflation of a nonlinear viscoelastic spherical membrane by monotonically increasing pressure is considered. The deformation is assumed to be spherically symmetric. For the constitutive equation assumed, circumstances are shown to exist when the radius history must either have a jump discontinuity or bifurcate. A necessary condition for bifurcation and its dependence on material properties and radius history is analysed. Examples of bifurcation for various pressure histories are presented. Post-bifurcation branches are constructed and the possibility of secondary bifurcation is discussed.
\end{abstract}

\section{INTRODUCTION}

A fascinating phenomenon in nonlinear elasticity arises in the problem of the inflation of a spherical membrane by internal pressure. Using the Mooney form of strain energy function and assuming spherically symmetric deformation Green and Shield[1] showed that there could be more than one equilibrium state corresponding to each internal pressure. This paper considers the analogous situation when the membrane material is viscoelastic. In particular, there may be a pressure history which generates several possible radius histories. An example is presented in which such pressure histories are constructed. The corresponding radius histories arise by bifurcation from a common history.

Consider first how bifurcation occurs in the elastic case. Starting from the undeformed state let the membrane be subjected to a continuous monotonically increasing pressure history. The deformation is considered quasi-static and can therefore be determined from the pressureradius relation obtained by Green and Shield. This relation is shown schematically in Fig. 2(a). As the internal pressure increases from zero, a continuous monotonically increasing radius history is found from the portion of the $f-x$ relation to the left of the local maximum. If the internal pressure, increases beyond this local maximum, the radius history has a jump discontinuity and then is found from the monotonically increasing portion of the $f-x$ relation to the right of the local minimum. Thus a jump discontinuity in radius history is produced even though the pressure history is continuous. In order to construct a continuous radius history. the pressure must be reduced after reaching the local maximum. The radius history then bifurcates into two continuous branches which are found by using the portions of the $f-x$ relation to either side of the local maximum.

There are several reasons for avoiding a jump discontinuity in the radius history. The internal pressure being greater than the local maximum of $f$ implies that the membrane wall force and pressure resultant are no longer in balance and inertial effects must be considered. The apparent size of the jump discontinuity suggests that inertial effects could be quite considerable. If the occurance of this phenomenon in polymer processing, such as blow forming, is considered, the motion is no longer under control. This is an undesirable situation since, for example, the sudden increase in radius could be so large that the corresponding reduction in wall thickness could lead to bursting.

When the membrane is viscoelastic, the above bifurcation analysis is complicated by history effects. The example presented here shows that the requirement of a continuous radius history can still lead to bifurcation. The question of restrictions on constitutive equations as related to the possibility of bifurcation is not considered. It is assumed that just as in the elastic case, there are material models for which bifurcation is possible. The example also includes the determination of the post-bifurcation solution branches corresponding to a given pressure history. The stability of such branches is not considered.

The governing equations are presented in Section 2 . The material is modeled by a nonlinear single integral constitutive equation which displays Mooney elasticity in its instantaneous and long time equilibrium response limits. Consequently, the response of the spherical membrane is 
described by a nonlinear single integral equation for the radius history, given the pressure history. A bifurcation condition for a general equation of this type is derived in Section 3. The solution procedure is outlined in Section 4. The problem is reformulated so that at each time it is analogous to the elastic problem described above. However, the form of the analogous $f-x$ relation from which the current radius is found from the current pressure depends on the past history. The properties of this relation and its variation with past history are discussed in Section 5, for the constitutive equation of Section 2. Using these properties, pre-bifurcation response is discussed in Section 6. Sections 7 and 8 are concerned with the construction of post-bifurcation branches. Numerical results are presented in Section 9. Certain results suggest an explanation for the apparent instability observed during the inflation of polymer fluid membranes. Other results indicate the complicated relation between material parameters, pressure histories and the possibility of bifurcation.

\section{FORMULATION}

Let the spherical membrane, in its undeformed state, have mean radius $a_{o}$ and wall thickness $h_{o}$. The membrane is assumed sufficiently thin, $h_{o} / a_{o} \ll 1$, so that the usual membrane approximations are valid. In particular, a physical variable has the same value on the mid-surface as at any point through the thickness. The deformation will be described with respect to a spherical polar coordinate system whose origin coincides with the center of the undeformed spherical membrane. In view of the symmetry of deformation, a particle of the membrane surface initially at $\left(a_{o}, \theta, \phi\right)$ moves radially to $\left(\rho\left(a_{o}, t\right), \theta, \phi\right)$ at time $t$. The principal stretch ratios in the membrane surface are equal and are denoted by

$$
\lambda_{\theta}\left(a_{o}, t\right)=\lambda_{\phi}\left(a_{o}, t\right)=\lambda\left(a_{o}, t\right)=\frac{\rho\left(a_{o}, t\right)}{a_{o}} .
$$

Letting $h(t)$ denote the wall thickness at time $t$, the radial stretch ratio is defined by the relation

$$
\lambda_{r}\left(a_{o}, t\right)=h\left(a_{o}, t\right) / h_{o}
$$

The membrane material is assumed incompressible so that at each time

$$
\lambda_{r}=\frac{1}{\lambda_{\theta} \lambda_{\phi}}=\frac{1}{\lambda^{2}} .
$$

Let $p(t)$ denote the internal pressure at time $t$. The principal stresses $\sigma_{\theta}$ and $\sigma_{\phi}$ are equal and are denoted by $\sigma(t)$, where dependence on initial radius $a_{o}$ is suppressed for notational convenience. Assuming quasi-static motion, the force balance condition becomes [2]

$$
p(t)=\frac{2 \sigma(t) h(t)}{\rho(t)} .
$$

Using (1)-(3), this becomes

$$
\frac{a_{o} p(t)}{2 h_{o}}=\frac{\sigma(t)}{\lambda^{3}(t)}
$$

The constitutive equations is one that was used in previous studies $[3,4]$ to illustrate a method for analyzing large non-homogeneous deformations of nonlinear viscoelastic membranes. Its tensorial form is

where

$$
\boldsymbol{\sigma}=-q \mathbf{1}+\mathbf{F} \mathscr{F} \mathbf{F}^{T},
$$

$$
\begin{gathered}
\mathscr{F}=\mathbf{R}[\mathbf{C}(t), 0]+\int_{0}^{t} \frac{\partial}{\partial(t-s)} \mathbf{R}[\mathbf{C}(s), t-s] \mathrm{d} s, \\
\mathbf{R}[\mathbf{C}(s), \xi]=R(\xi)\{[1+\alpha I(s)] \mathbf{1}-\alpha \mathbf{C}(s)\}, \\
R(\xi)=C_{0}\left[(1-\gamma) \mathrm{e}^{-\xi / \tau_{R}}+\gamma\right], \quad \alpha>0, \quad 0<\gamma<1 .
\end{gathered}
$$


In the above, $q$ is an arbitrary scalar arising from the incompressibility constraint, 1 denotes the unit tensor, $\mathbf{F}$ denotes the current deformation gradient $\left(\mathbf{F}=\left[\partial x_{i} / \partial X_{j}\right]\right.$ in Cartesian coordinates, with current coordinates $x_{i}=x_{i}\left(X_{j}, t\right)$ and reference coordinates $\left.X_{j}\right), \mathbf{C}(s)=\mathbf{F}(s)^{T} \mathbf{F}(s)$, and $I$ is the first stretch ratio invariant which is expressed in terms of the principal stretch ratios as

$$
I=\lambda_{r}^{2}+\lambda_{\theta}^{2}+\lambda_{\phi}^{2}
$$

Expressing (6) relative to the principal directions, and eliminating $q$ on the basis that $\sigma_{\rho}(t)$ is small compared to $\sigma(t)$, the constitutive equation for equal biaxial stretching in the spherical membrane surface reduces to

$$
\sigma(t)=C_{n}\left(\lambda^{2}-\frac{1}{\lambda^{4}}\right)\left(1+\alpha \lambda^{2}\right)+\int_{0}^{t} \frac{\partial R(t-s)}{\partial(t-s)}\left[\left(\lambda^{2}-\frac{1}{\lambda^{4}}\right)+\alpha\left(\lambda^{2} \lambda^{2}(s)-\frac{2 \lambda^{2}(s)}{\lambda^{4}}+\frac{\lambda^{2}}{\lambda^{4}(s)}\right)\right] \mathrm{d} s,
$$

where $\lambda=\lambda(t)$.

This describes a material whose instantaneous response is the same as a nonlinear elastic solid of Mooney type, with Mooney parameter $\alpha$ (dimensionless) and elastic parameter $C_{o}$ (dimensions of stress). Its long time equilibrium response also duplicates a Mooney elastic material, with the same Mooney parameter, but with elastic parameter $\gamma C_{o}$. In a step stretch test, the stress relaxes exponentially with a single relaxation time $\tau_{R}$. This model incorporates all essential features of nonlinear viscoelastic solid behavior. Since the short time response is like that of a Mooney material, which is known to exhibit bifurcation of response, this model should allow the influence of viscoelasticity to be assessed.

Substituting (7) into (5) gives the governing equation for the response of a viscoselastic spherical membrane

$$
P(t)=\frac{1}{\lambda}\left(1-\frac{1}{\lambda^{6}}\right)\left(1+\alpha \lambda^{2}\right)+\frac{1}{\lambda} \int_{0}^{t} \frac{\partial R(t-s)}{\partial(t-s)}\left[\left(1-\frac{1}{\lambda^{6}}\right)+\alpha\left(\lambda^{2}(s)-\frac{2 \lambda^{2}(s)}{\lambda^{6}}+\frac{1}{\lambda^{4}(s)}\right)\right] \mathrm{d} s,
$$

where

$$
P(t)=\frac{a_{o} p(t)}{2 h_{o} C_{o}}
$$

\section{BIFURCATION CONDITION}

For convenience, rewrite ( 8 ) as

$$
P(t)=A(\lambda(t))+\int_{0}^{t} B(\lambda(t), \lambda(s), t-s) \mathrm{d} s
$$

Assume that the membrane is initially undeformed and that $\lambda(t)$ is a continuous function of $t$. Hence $\lambda(0)=1$ and it follows from (8) that $P(0)=0$. Let $P(t)$ be a monotonically increasing continuous function of $t$, and assume that (8) has a unique solution $\lambda(t)$ for $0 \leq t \leq T^{*}$. Let the solution bifurcate at time $T^{*}$ and denote the branches by $\lambda(t)$ and $\lambda(t)+\Delta \lambda(t), T^{*} \leq t$ (see Fig. 1). $\Delta \lambda(t)$ is a continuous function which satisfies

$$
\begin{array}{cc}
\Delta \lambda(t)=0, & 0 \leq t \leq T^{*} \\
\neq 0, & T^{*}<t .
\end{array}
$$

For some $\Delta t>0$,

$$
\begin{aligned}
P\left(T^{*}+\Delta t\right)= & A\left(\lambda\left(T^{*}+\Delta t\right)\right)+\int_{0}^{T^{*}+\Delta t} B\left(\lambda\left(T^{*}+\Delta t\right), \lambda(s), T^{*}+\Delta t-s\right) \mathrm{d} s \\
= & A\left(\lambda\left(T^{*}+\Delta t\right)+\Delta \lambda\left(T^{*}+\Delta t\right)\right)+\int_{0}^{T^{*}+\Delta T} B\left(\lambda\left(T^{*}+\Delta t\right)+\Delta \lambda\left(T^{*}+\Delta t\right),\right. \\
& \left.\lambda(s)+\Delta \lambda(s), T^{*}+\Delta t-s\right) \mathrm{d} s .
\end{aligned}
$$




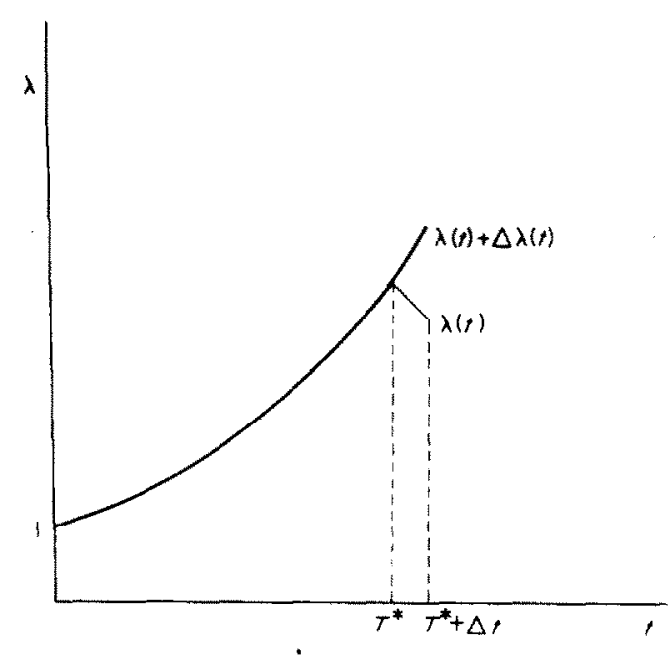

Fig. 1. Branching of the stretch ratio history at time $T^{*}$.

For sufficiently small $\Delta t$ and assuming appropriate smoothness for $A$ and $B$, it follows from (11) and (10) that

$$
\begin{aligned}
\left\{\frac{\partial A}{\partial \lambda}\left(\lambda\left(T^{*}+\Delta t\right)\right)+\int_{0}^{T^{*}+\Delta t} \frac{\partial B}{\partial \lambda(t)}\left(\lambda\left(T^{*}+\Delta t\right), \lambda(s), T^{*}+\Delta t-s\right) \mathrm{d} s\right\} \Delta \lambda\left(T^{*}+\Delta t\right) \\
+\int_{T^{*}}^{T^{*}+\Delta t} \frac{\partial B}{\partial \lambda(s)}\left(\lambda\left(T^{*}+\Delta t\right), \lambda(s), T^{*}+\Delta t-s\right) \Delta \lambda(s) \mathrm{d} s+o(\overline{\Delta \lambda})=0 .
\end{aligned}
$$

where

$$
\overline{\Delta \lambda}=\max |\Delta \lambda(s)|, \quad s \epsilon\left[T^{*}, T^{*}+\Delta t\right] .
$$

Divide through (12) by $\Delta t$ and consider the limit as $\Delta t \rightarrow 0$. By (10),

$$
\begin{gathered}
\lim _{\Delta t \rightarrow 0} \frac{1}{\Delta t} \int_{T^{*}}^{T^{*}+\Delta T} \quad \frac{\partial B}{\partial \lambda(s)}\left(\lambda\left(T^{*}+\Delta t\right), \lambda(s), T^{*}+\Delta t-s\right) \Delta \lambda(s) \mathrm{d} s \\
=\frac{\partial B}{\partial \lambda(s)}\left(\lambda\left(T^{*}\right), \lambda\left(T^{*}\right), 0\right) \Delta \lambda\left(T^{*}\right)=0 .
\end{gathered}
$$

Defining

$$
\lim _{\Delta t \rightarrow 0} \frac{\Delta \lambda\left(T^{*}+\Delta t\right)}{\Delta t}=\hat{\lambda}\left(T^{*}\right)
$$

(12) reduces in the limit to

$$
\left\{\frac{\partial A}{\partial \lambda}\left(\lambda\left(T^{*}\right)\right)+\int_{0}^{T^{*}} \frac{\partial B}{\partial \lambda(t)}\left(\lambda\left(T^{*}\right), \lambda(s), T^{*}-s\right) \mathrm{d} s\right\} \hat{\lambda}\left(T^{*}\right)=0
$$

Thus, a necessary condition that the solution bifurcate at time $T^{*}$, i.e. $\hat{\lambda}\left(T^{*}\right) \neq 0$, is that

$$
\left.\frac{\partial}{\partial \lambda(t)}\left\{A(\lambda)+\int_{0}^{T^{*}} B\left(\lambda(l), \lambda(s), T^{*}-s\right) \mathrm{d} s\right\}\right|_{\lambda(t)=\lambda\left(T^{*}\right)}=0 .
$$

Condition (14) has two interpretations. If the bifurcation time $T^{*}$ is specified, then (14) acts as a constraint on solutions of (9). Conversely, if a solution to (9) is being constructed, then the left hand side of (14) acts as a side condition for determining whether the solution is approaching possible bifurcation. This is discussed in the next section. 


\section{ANALYTICAL DISCUSSION}

The solution of (9) can be illustrated graphically. Suppose the stretch ratio history previous to time $t, \lambda(s), s \in[0, t)$, has been found. Based on the right hand side of (9), define the response function

$$
f(x, t)=A(x)+\int_{0}^{t} B(x, \lambda(s), t-s) \mathrm{d} s,
$$

whose dependence on $x$ is clearly influenced by past history. Then, according to $(9), \lambda(t)$ is the solution to the equation

$$
P(t)=f(x, t)
$$

In this way, the determination of the stretch ratio history can be represented graphically as in Fig. 3. This construction procedure also allows a convenient graphical interpretation for bifurcation condition (14). In view of (15), this can be expressed as

$$
\frac{\partial}{\partial x} f\left(\lambda\left(T^{*}\right), T^{*}\right)=0
$$

Thus, the bifurcation condition can be satisfied at time $t$ if the solution of (16) lies at a local maximum of $f(x, t)$ with respect to $x$. At other times $t^{\prime}$, the left hand side of (14) or (14a) gives the slope of $f\left(x, t^{\prime}\right)$ at the solution of (16). A decrease in its magnitude as time increases indicates impending bifurcation.

The satisfaction of the bifurcation condition depends on whether $f(x, t)$ has a local maximum in $x$. For the particular model presented in Section 2, the $x$ dependence of $f(x, t)$ at fixed $t$ and its change with time can be discussed in some detail. To this end, let (8) be rewritten as

$$
P(t)=\frac{C(t)}{\lambda}-\frac{D(t)}{\lambda^{\top}}+\alpha \lambda^{2}\left(\frac{1}{\lambda}-\frac{1}{\lambda^{7}}\right)
$$

where

$$
\begin{aligned}
& C(t)=R(t)+\alpha \int_{0}^{t} \dot{R}(t-s)\left(\lambda^{2}(s)+1 / \lambda^{4}(s)\right) \mathrm{d} s \\
& D(t)=R(t)+2 \alpha \int_{0}^{t} \dot{R}(t-s) \lambda^{2}(s) \mathrm{d} s
\end{aligned}
$$

$\dot{R}(s)=\mathrm{d} R(s) / \mathrm{d} s$, and $R(\xi)=(1-\gamma) \mathrm{e}^{-\xi}+\gamma$, following non-dimensionalization.

Response function (15) for this case becomes

$$
f(x, t)=\frac{C(t)}{x}-\frac{D(t)}{x^{7}}+\alpha x^{2}\left(\frac{1}{x}-\frac{1}{x^{7}}\right)
$$

At each time, coefficients $C(t)$ and $D(t)$ are determined by the previous solution history. As they vary with time, so does the form of $f(x, t)$.

The specific form of $f(x, t)$ given by (17a) and (18) allows its dependence on $x$ and $t$ to be studied in some detail. It is shown that for sufficiently small $\alpha, f(x, t)$ starts off with the form in Fig. 2(a). As time increases, it tends to flatten out and may evolve into the form in Fig. 2(b). These results are developed in the next sections.

\section{PROPERTIES OF $f(x, t)$}

The dependence of $f(x, t)$ on $x$ for fixed $t$ is discussed in (i)-(iv) below. Let $t(\alpha)>0$ denote a time depending on $\alpha$. Each result below is valid in a different time interval $[0, t(\alpha)]$. In order 


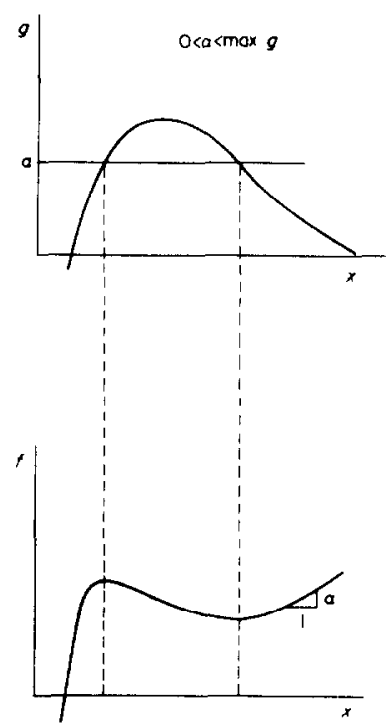

(o)
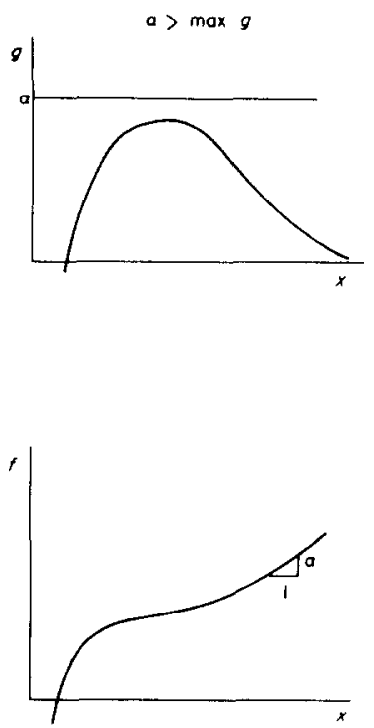

(b)

Fig. 2. Dependence of the form of $f(x, t)$ on the relative values of $\alpha$ and $g(x, t)$; (a) 2 positive real roots, (b) 0 positive real roots.

to avoid cumbersome notation reference to $t(\alpha)$ is omitted but understood, except when the result is valid for all $t \geq 0$. Note that $R(t)>0, \dot{R}(t)<0, \lambda(t) \geq 1$.

(i) Since the leading terms in (17a) are positive and the integrals are negative $C(t)>0$, $D(t)>0$ in some time interval. It can also be seen that $D(t)-C(t) \leq 0$, for $t \geq 0$.

(ii) From (18) and (i),

$$
\begin{gathered}
f(1, t)=C(t)-D(t) \geq 0, t \geq 0, \\
f(x, t) \approx-D(t) / x^{7} \rightarrow-\infty \text { as } x \rightarrow 0, \\
f(x, t) \approx \alpha x \text { as } x \rightarrow \infty, t \geq 0 .
\end{gathered}
$$

(iii) From (17a) and (18)

$$
\begin{aligned}
\frac{\partial f}{\partial x}(x, t) & =f_{x}(x, t)=\left(\alpha x^{8}-C(t) x^{6}+5 \alpha x^{2}+7 D(t)\right) / x^{8} \\
& =\frac{R(t)}{x^{8}}\left(7-x^{6}\right)+\alpha\left(1+\frac{5}{x^{6}}\right)+\frac{\alpha}{x^{8}} \int_{0}^{t} \dot{R}(t-s)\left[14-x^{6}\left(1+\frac{1}{\lambda^{6}(s)}\right)\right] \lambda^{2}(s) \mathrm{d} s .
\end{aligned}
$$

For each $\alpha$ there is a time interval and an $x$-interval, $1 \leq x \leq \bar{x}(t, \alpha), \bar{x}(t, \alpha)>0$, in which the leading terms dominate and $f_{x}(x, t)>0$. Also

$$
\begin{gathered}
f_{x}(x, t) \approx 7 D(t) / x^{8} \rightarrow \infty \quad \text { as } \quad x \rightarrow 0, \\
f_{x}(x, t) \rightarrow \alpha \quad \text { as } \quad x \rightarrow \infty, \quad t \geq 0 .
\end{gathered}
$$

Thus, $f_{x}(x, t)$ has positive slope as $x \rightarrow 0$, in a neighborhood to the right of $x=1$, at least initially, and as $x \rightarrow \infty$.

(iv) The following analysis is based on that of Green and Shield [1] as presented in Green and Adkins ([2], p. 161). For $f(x, t)$ to have a local maximum, then by (19) there is some $x$ for which

$$
\alpha x^{8}-C(t) x^{6}+5 \alpha x^{2}+7 D(t)=0
$$


Assume $\alpha>0$ and $C(t)>0, D(t)>0$ for the present. Applying Descartes' rule of signs [5], (20) has 2 sign alternations and therefore either 0 or 2 positive real roots. In view of results (iii), if there are no positive real roots, then $f_{x}(x, t)>0,0<x<\infty$. If there are 2 positive real roots, $f_{x}(x, t)<0$ for $x$ in some bounded subinterval of $[0, \infty)$. When $f_{x}(1, t)>0$, these roots correspond to a local maximum and minimum of $f(x, t)$, both roots being either less than $x=1$ or both greater. $f(x, 0)$ coincides with the function discussed in Green and Adkins [2]. It was shown there that when $0<\alpha<0.21, f_{x}(x, 0)$ has 2 roots which are both greater than $x=1$. The same will be expected when $t>0$.

A necessary condition that (20) have 2 positive real roots is obtained from (19) and the requirement that $f_{x}(x, t)<0$ on some interval of $x$. It is the following inequality:

$$
\alpha<\frac{C(t) y^{3}-7 D(t)}{y^{4}+5 y}=g(y, t), \text { say, }
$$

where $y=x^{2}$. To determine when (21) is met, it is necessary to study the properties of $g(y, t)$. First, note that

$$
\frac{\partial g}{\partial y}(y, t)=g_{y}(y, t)=\frac{-C(t) y^{6}+(10 C(t)+28 D(t)) y^{3}+35 D(t)}{\left(y^{4}+5 y\right)^{2}} .
$$

Then, by a discussion similar to the above, it can be shown that for some time interval

$$
\begin{gathered}
g(1, t)=-(7 D(t)-C(t)) / 6<0, \quad g_{y}(1, t)=(9 C(t)+63 D(t)) / 36>0 \\
g(y, t) \approx C(t) / y>0, \quad g_{y}(y, t) \approx-C(t) / y^{2}<0, \quad y>1
\end{gathered}
$$

and $g_{y}(y, t)$ has a single positive real root. Thus, $g(y, t)$ has a local maximum at $y_{m}(t)$ given by

$$
y_{m}{ }^{3}(t)=5+14 D / C+\left[(5+14 D / C)^{2} \times 35 D / C\right]^{1 / 2} .
$$

For sufficiently small $t, D(t) / C(t) \approx 1$ and $y_{m}(t)$ is much greater than 1 . If $(21)$ is met, this occurs for $y($ or $x)>1$. The two roots of $f_{x}$ are thus greater than one and $f(x, t)$ has a local maximum for $x>1$, the physically meaningful range. It remains to be determined whether $g\left(y_{m}(t), t\right)$ is sufficiently large that (21) is met. The possibilities are illustrated in Fig. 2. Which situation arises depends on $\alpha$ and the interaction of previous stretch ratio history and relaxation properties as reflected in the values of $C(t)$ and $D(t)$.

When $\alpha=0$, (17a) shows that $C(t)=D(t)=R(t)$. (20) has the single root $x=7^{1 / 6}>1$, $t \geq 0$. It follows from the above results that for $t \geq 0, f(x, t)$ has only a local maximum with $f(1, t)=0$ and $f(x, t) \rightarrow 0$ as $x \rightarrow \infty$. The properties of $g(y, t)$ are not needed in this case.

The change of $f(x, t)$ as $t$ increases is discussed in (v)-(viii) next. Attention is confined to stretch ratio histories for which $\dot{\lambda}(s)>0$.

(v) Integrating $C$ and $D$ in (17a) by parts and then differentiating gives

$$
\begin{aligned}
& \dot{C}=\dot{R}(1+2 \alpha)+\alpha \int_{0}^{t} \dot{R}(t-s)\left(2 \lambda(s)-4 / \lambda^{s}(s)\right) \dot{\lambda}(s) \mathrm{d} s, \\
& \dot{D}=\dot{R}(1+2 \alpha)+\alpha \int_{0}^{t} \dot{R}(t-s) 4 \lambda(s) \dot{\lambda}(s) \mathrm{d} s,
\end{aligned}
$$

It follows that $\dot{D}(t)-\dot{C}(t) \leq 0, \dot{D}(t) \leq 0, t \geq 0$ and $\dot{C}(t) \leq 0$ for some time interval.

(vi) From (17a) and (18).

$$
\frac{\partial f(x, t)}{\partial t}=f_{t}(x, t)=\dot{R}(1+2 \alpha)\left(1-\frac{1}{x^{6}}\right) \frac{1}{x}+\alpha \int_{0}^{t} \dot{R}(t-s)\left[1-\frac{2}{x^{6}}-\frac{2}{\lambda^{6}(s)}\right] \frac{2 \lambda(s) \dot{\lambda}(s)}{x} \mathrm{~d} s .
$$

For $t \geq 0, f_{t}(1, t) \geq 0$. For each $\alpha$, there is a time interval and an $x$-interval, $1<\hat{x}(t, \alpha) \leq x<$ $\infty$, in which the leading term dominates and $f_{t}(x, t)<0$. The integral also becomes negative for 
sufficiently large $\lambda(s)$ and $x$. When $\alpha=0, f_{t}(1, t)=0$ and $f_{t}(x, t)<0, x>1, t \geq 0$. Thus, except in a neighborhood of $x=1, f(x, t)$ decreases with $t$.

(vii) By (17a), $C(0)=D(0)=1$. According to the discussion in Green and Adkins [2], $\max g(y, 0)=0.21$. Thus, for $0 \leq \alpha<0.21, f(x, 0)$ is as shown in Fig. 2(a). For such $\alpha$, there is a time interval in which $g\left(y_{m}(t), t\right)>\alpha$ and $f(x, t)$ has a local maximum. Let $x_{m}(t)$ be the root of (20) corresponding to this local maximum, and define $f_{m}(t)=f\left(x_{m}(t), t\right)$ and $f_{m}=\mathrm{d} f_{m} / \mathrm{d} t$. Then, since $f_{x}\left(x_{m}(t), t\right)=0$,

$$
\hat{f}_{m}(t)=\dot{R}(1+2 \alpha)\left(1-\frac{1}{x_{m}^{6}}\right) \frac{1}{x_{m}}+\alpha \int_{0}^{t} \dot{R}(t-s)\left[1-\frac{2}{x_{m}^{6}}-\frac{2}{\lambda^{6}(s)}\right] \frac{2 \dot{\lambda}(s) \lambda(s)}{x_{m}} \mathrm{~d} s .
$$

Letting $x_{m}(t, \alpha)$ denote a root of $(20)$, then $x_{m}(t, 0)=7^{1 / 6}$. If the variation of $x_{m}(t, \alpha)$ with $\alpha$ is sufficiently smooth, then there is some time interval in which $x_{m}(t, \alpha) \approx 7^{1 / 6}$. The leading term of (25) dominates and $\dot{f}_{m}(t)<0$. When $\alpha=0, \dot{f}_{m}(t)=R\left(1-7^{-1}\right) 7^{-1 / 6}<0$, the local maximum of $f(x, t)$ decreases with $t$.

(viii) Recalling $y_{m}(t)$ given by (22), define $g_{m}(t)=g\left(y_{m}(t), t\right)$ and $\dot{g}_{m}=\mathrm{d} g_{m} / \mathrm{d} t$. Then using (21), (23) and proceeding as in (vii)

$$
\dot{g}_{m}(t)=\frac{1}{y_{m}{ }^{4}+5 y_{m}}\left\{\dot{R}(1+2 \alpha)\left(y_{m}{ }^{3}-7\right)+\alpha \int_{0}^{t} \dot{R}(t-s)\left(2 \lambda(s)\left(y_{m}{ }^{3}-14\right)-\frac{4 y_{m}{ }^{3}}{\lambda^{3}(s)}\right) \dot{\lambda}(s) \mathrm{d} s\right\} .
$$

By (22) there is some time interval in which $y_{m}{ }^{3}(t)$ is sufficiently large that $\dot{g}_{m}(t)<0$.

\section{PRE-BIFUCATION SOLUTION OF (16), (18)}

The behavior of (16) and (18) as the solution approaches befucation can now be discussed. Consider continuous pressure histories with $\dot{P}(t)>0$ and continuous stretch ratio histories $\lambda(t)$, with $\lambda(0)=1$. It is first shown that $\lambda(t)$ monotonically increases in some time interval.

Since $\lambda(t)$ satisfies (16),

$$
\dot{P}(t)=f_{x}(\lambda(t), t) \dot{\lambda}(t)+f_{t}(\lambda(t), t)
$$

By (iii), $f_{x}(1,0)>0$ and by (vi) $f_{t}(1,0)=0$ so that

$$
\dot{\lambda}(0)=\dot{P}(0) \mid f_{x}(1,0)=\frac{\dot{P}(0)}{6(1+\alpha)}>0 .
$$

For some small time interval (26) implies $\lambda(t)=1+\dot{\lambda}(0) t+O\left(t^{2}\right)$. Using this and neglecting terms of $0\left(t^{2}\right)$, it can be shown from (24) that $f_{t}(\lambda(t), t)=\dot{R}(0) \dot{P}(0) t<0$. Together with (vi), this implies $f_{t}(\lambda(t), t)<0$ for some time interval. Since (iii) implies that $f_{x}(\lambda(t), t)>0$, it follows from (26) that $\dot{\lambda}(t)>0$. Furthermore, by (19) and (24), when $\alpha=0$, it follows from (26) that $\dot{\lambda}(t)>0$ as long as $\lambda(t)<7^{1 / 6}$. This suggests that for each $\alpha>0$, there is some time interval in which $\dot{\lambda}(t)>0$.

Alternatively, when $\dot{P}(t)>0$, there is some time interval in which (16) has a continuous monotonically increasing solution $\lambda(t)$ determined by $f(x, t), 1 \leq x \leq x_{m}(t)$. If $0 \leq \alpha \leq 0.21$, result (iv) shows that $f(x, t)$ has a local maximum and minimum as in Fig. 2(a). As the solution progresses $f_{m}(t)$ monotonically decreases (result (vii)) and, except for a neighborhood of $x=1$, so does $f(x, t)$, (result (vi)). A graphical representation is shown in Fig. 3.

A continuous solution can be constructed in this way as long as

$$
P(t) \leq f\left(x_{m}(t), t\right)=f_{m}(t) .
$$

However, this condition may cease to be satisfied. If $\alpha=0, \lambda(t)$ increases and $f_{m}(t)$ decreases as long as $\lambda(t)<7^{1 / 6}$. Since $f_{m}(t)$ occurs at $x=7^{1 / 6}$, a time $T^{*}$ always exists such that

$$
P\left(T^{*}\right)=f\left[x_{m}\left(T^{*}\right), T^{*}\right] .
$$



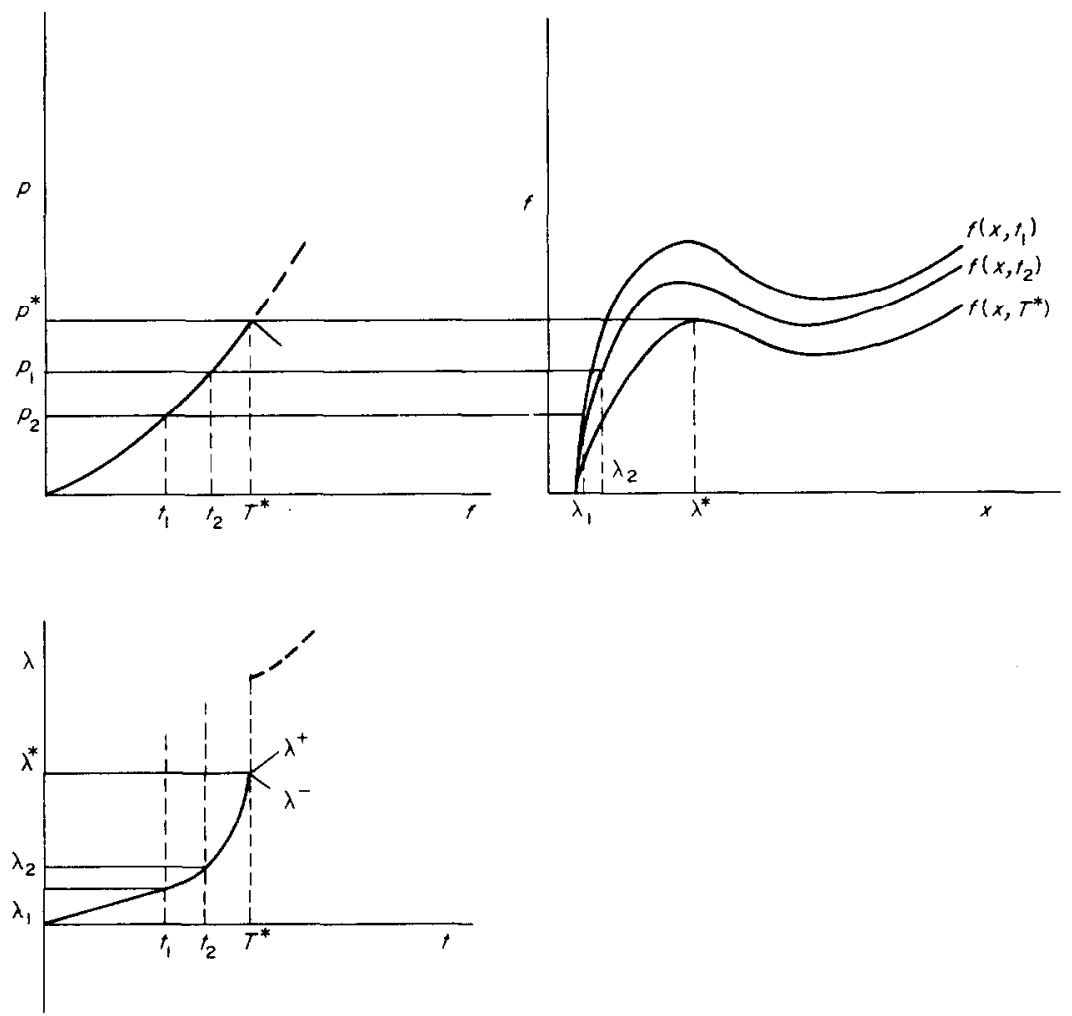

Fig. 3. Graphical representation of the construction of stretch ratio history $\lambda(t)$ up to bifurcation.

For $0<\alpha<0.21$, the time interval in which $\lambda(t)$ increases and $f_{m}(t)$ decreases may be long enough that (29) is met. On the other hand, since $\dot{g}_{m}(t)<0$ (by (viii)), the situation is approaching that in Fig. 2(b). $f(x, t)$ becomes monotonic and the possibility for bifurcation vanishes. For the present, it will be assumed that (29) is satisfied.

For $t>T^{*}$, if $P(t)>P\left(T^{*}\right)$, then $P(t)>f\left(x_{m}(t), t\right)$. The only solution to $(16)(\alpha>0)$ will lie on the monotonically increasing part of $f(x, t)$ beyond the local minimum, thereby inducing a jump discontinuity in the stretch ratio history (see Fig. 3). If a continuous solution is to be maintained, then $P(t)$ must decrease for $t>T^{*}$.

A unique continuous continuation to $\lambda(s), 0<s<T^{*}$, can be obtained if

$$
P(t)=f\left(x_{m}(t), t\right), \quad t \geq T^{*},
$$

which is $\lambda(t)=x_{m}(t), t \geq T^{*}$. On the other hand, if $P(t)$ is decreased faster than $f\left(x_{m}(t), t\right)$, then two continuous continuations can be found, $\lambda^{-}(s)$ and $\lambda^{+}(s)$, determined by solutions of (18) for $1 \leq x \leq x_{m}(t)$ and $x_{m}(t) \leq x$, respectively.

In this way, $T^{*}$ is seen to be a bifurcation time, and the existence of a local maximum of $f(x, t)$ is seen to be a necessary condition for bifurcation. This latter is equivalent to condition (14a).

\section{POST-BIFURCATION RESPONSE}

The construction of branches following bifurcation leads to a number of interesting complications. For the present, let $f(x, t)$ be as depicted in Fig. 2(a). Discussion as to how this form changes will be given in Section 8 .

If $P(t)$ decreases faster than $f\left(x_{m}(t), t\right), t>T^{*}$, two stretch ratio histories are generated, $\lambda^{-}(s)$ and $\lambda^{+}(s)>\lambda^{-}(s)$, as discussed above. Two sets of coefficients (17a) are generated, $\left(C^{-}(t), D^{-}(t)\right)$ from $\lambda^{-}(s)$ and $\left(C^{+}(t), D^{+}(t)\right)$ from $\lambda^{+}(s), 0 \leq s \leq t$. By (18), this generates two response functions $f^{-}(x, t)$ and $f^{+}(x, t)$. These are assumed to have the same form as the prebifurcation $f(x, t)$, at least for $t$ near $T^{*}$, with local maxima at $x_{m}{ }^{-}(t), x_{m}{ }^{+}(t)$, respectively. 
Let $x_{\min }^{-}(t)$ and $x_{\min }^{+}(t)$ denote the abscissae of their local minima. Then $\lambda^{-}(t)$ is the solution of

$$
P(t)=f^{-}(x, t) \text { on } \quad 1 \leq x \leq x_{m}^{-}(t)
$$

and $\lambda^{+}(t)$ is the solution

$$
P(t)=f^{+}(x, t), \quad \text { on } x_{m}^{+}(t) \leq x \leq x_{\min }^{+}(t) .
$$

The separation of $f(x, t)$ into response functions for each solution branch can be determined. Let $\lambda_{m}{ }^{o}(t)=x_{m}{ }^{o}(t)$ be the solution of (30), i.e. the solution obtained when $P(t)$ follows the local maximum of $f(x, t), t>T^{*}$. Denote the response function based on this history as $f^{o}(x, t)$ and introduce the variations

$$
\begin{gathered}
\delta \lambda^{*}(t)=\lambda^{*}(t)-\lambda_{m}{ }^{o}(t), \quad \delta x_{m}^{*}(t)=x_{m}^{*}(t)-x_{m}{ }^{o}(t), \quad t \geq T^{*}, \\
\delta \lambda^{*}(t)=\delta x_{m}^{*}(t)=0, \quad 0 \leq t \leq T^{*}, \\
\delta f_{m}^{*}(t)=f_{m}^{*}\left(x_{m}^{*}(t), t\right)-f_{m}^{o}\left(x_{m}(t), t\right),
\end{gathered}
$$

where ${ }^{*}$ denotes + or - . Then the variation $\delta f_{m}^{*}(t)$ due to the variation in stretch ratio history $\delta \lambda^{*}(t)$ can be shown to have form.

$$
\delta f_{m}^{*}(t)=\alpha \int_{0}^{t} \dot{R}(t-s)\left[1-\frac{2}{\left(\lambda_{m}{ }^{o}(s)\right)^{6}}-\frac{2}{\left(x_{m}{ }^{o}(t)\right)^{6}}\right] \frac{2 \lambda_{m}{ }^{o}(s)}{x_{m}{ }^{o}(t)} \delta \lambda^{*}(s) \mathrm{d} s .
$$

By result (iv) of Section 4, when $\alpha=0, \lambda_{m}{ }^{\circ}(s)=x_{m}{ }^{o}(s)=7^{1 / 6}$. If it is assumed that this is a reasonable approximation for some $\alpha>0$, then the quantity in the square brackets is positive. Numerical results support this assumption. Since $\dot{R}(t)<0$, there is some time interval following $T^{*}$, in which

$$
\begin{array}{lll}
\delta f_{m}{ }^{-}(t)>0 & \text { for } & \delta \lambda^{*}(s)=\delta \lambda^{-}(s)<0 \\
\delta f_{m}{ }^{+}(t)<0 & \text { for } & \delta \lambda^{*}(s)=\delta \lambda^{+}(s)>0 .
\end{array}
$$

For fixed $x$, the variation $\delta f^{*}(x, t)=f^{*}(x, t)-f^{o}(x, t)$ has the same form as (32) with $x_{m}{ }^{o}(t)$ replaced by $x$. Except for some neighborhood of $x=1$, a similar discussion suggests that (33) is also true for $\delta f^{*}(x, t)$ (see Fig. 4). It follows that a necessary condition for two continuous branches to be generated is

$$
P(t)<f^{+}\left(x_{m}^{+}(t), t\right)
$$

\section{Comment 1}

Let $f^{+}(x, t)$ have a local minimum at $x_{\min }^{+}(t)$. If $P(t)<f^{+}\left(x_{\min }^{+}(t), t\right)$, then a continuous

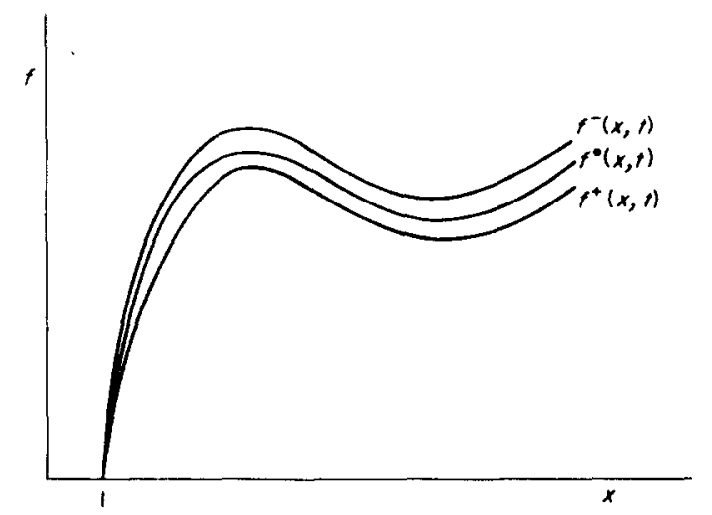

Fig. 4. Response functions for each branch following bifurcation. 
continuation of $\lambda^{+}(t)$ no longer exists. With this and (34), it is seen that upper and lower bounds on $P(t)$ are needed in order that $\lambda^{-}(t)$ and $\lambda^{+}(t)$ have simultaneous continuous extensions.

\section{Comment 2}

Suppose for some time $T^{* *}>T^{*}, P(t)$ coincides with $f^{+}\left(x_{m}{ }^{+}(t), t\right)$ or $f^{+}\left(x_{\min }^{+}(t), t\right)$. A repetition of the preceding discussion shows that there can be secondary bifurcation from the solution branch $\lambda^{+}(t)$ at time $T^{* *}$. This suggests that pressure histories $P(t)$ can be constructed for which any number of bifurcations can be produced at arbitrary times.

\section{CHANGE OF FORM OF RESPONSE FUNCTIONS}

To this point, it has been assumed that in the time intervals of concern, the response functions $f(x, t)$ have a local maximum and a local minimum. The time dependence of $C$ and $D$ in (17) allows the structure of $f(x, t)$ to vary significantly. Recall result (viii), Section 4 , that $\dot{g}_{m}(t)<0$. If $g_{m}(t)>\alpha$ initially, so that $f(x, t)$ has the form shown in Fig. 2(a), then as $t$ increases it may happen that $g_{m}(t)<\alpha$ and $f(x, t)$ becomes monotonic as in Fig. 2(b). Suppose this happens at time $\tau$. If $P(t)$ increases sufficiently slowly, then for $t>\tau, f_{x}(x, t)>0,1 \leq x<\infty$, and the bifurcation conditions (14a) or (29) will never be met. The alternative, that this condition is met before $f(x, t)$ becomes monotonic, will be discussed first.

The preceding discussion on the evolution of $f(x, t)$ to monotonicity also applies to $f^{o}(x, t)$, $f^{-}(x, t)$ and $f^{+}(x, t)$. Let $g^{o}(y, t), g^{-}(y, t)$ and $g^{+}(y, t)$ denote their respective condition functions for negative slopes. Denote the locations of their respective maxima by $y_{m}{ }^{o}(t), y_{m}{ }^{-}(t), y_{m}{ }^{+}(t)$, respectively. Then in some interval $\left[T^{*}, \bar{T}\right]$, the variation $\delta g_{m}^{*}(t)=g^{*}\left(y_{m}^{*}(t), t\right)-g^{o}\left(y_{m}{ }^{o}(t), t\right)$ due to variation $\delta \lambda^{*}(t)$, defined in (31), can be obtained by an argument similar to that in Section 7. It can be shown that

$$
\begin{array}{lll}
\delta g_{m}{ }^{-}(t)>0 & \text { for } & \delta \lambda^{*}(s)=\delta \lambda^{-}(s)<0, \\
\delta g_{m}{ }^{+}(t)<0 & \text { for } & \delta \lambda^{*}(s)=\delta \lambda^{+}(s)>0 .
\end{array}
$$

It may happen that $g^{+}\left(y_{m}{ }^{+}(t), t\right)<\alpha$ while $g^{-}\left(y_{m}{ }^{-}(t), t\right)>\alpha$, which means that $f^{+}(x, t)$ becomes monotonic before $f^{-}(x, t)$.

\section{Comment 3.}

The restrictions on $P(t)$ referred to in Comment 1 no longer apply. If $P(t)$ is now free to coincide with the local maximum of $f^{-}(x, t)$ at some time $T^{* * *}$, this becomes a bifurcation time for new solution branches for $\lambda^{-}(t)$.

$f(x, t)$, (or $f^{*}(x, t)$ following bifurcation) can also change form due to sign changes of $C$ or $D$. This possibility is indicated by result $(v)$, that they monotonically decrease. Two possible cases arise:

(a) $D<0, C<0$ will occur first. Condition (20) for local maxima and minima of $f(x, t)$ becomes

$$
\alpha x^{8}-C(t) x^{6}+5 \alpha x^{2}-7|D(t)|=0 .
$$

The three sign alternations imply that there are either 1 or 3 positive real roots. The possible forms for $f(x, t)$ are shown by Fig. 5 but will not be discussed further here.

(b) $D<0, C<0$, Condition (20) becomes

$$
\alpha x^{8}+|C(t)| x^{6}+5 \alpha x^{2}-7|D(t)|=0 .
$$

which has only 1 positive real root. $f(x, t)$ must have the form shown in Fig. S(b).

Suppose the response functions evolve into either of these forms as the solution branches are being determined. The value of $\lambda^{-}(t)$ or $\lambda^{+}(t)$ relative to the abscissae of the local maximum or local minimum must be considered in order to construct continuous extensions. For example, it might be anticipated that if $f^{-}$or $f^{+}$has the form indicated in Fig. 5(b), then $\lambda^{-}>x_{\min }^{-}$and $\lambda^{+}>x_{\min }^{+}$. If $f^{-}$or $f^{+}$has the form indicated in Fig. 5a, it is likely that $x_{\min 1}<\lambda^{-}<x_{\max }^{-}$and $x_{\max }^{+}<\lambda^{+}<x_{\min r}^{+}$ 


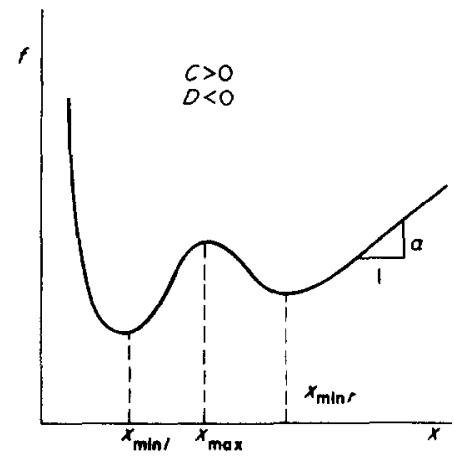

(a)

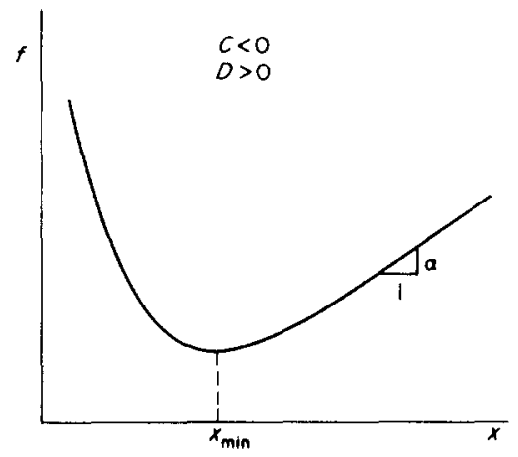

(b)

Fig. 5. Dependence of the forms of $f(x, t)$ on the stretch ratio history; (a) $C>0, D<0$; (b) $D<0, C<0$.

\section{Comment 4.}

From (17), the instantaneous elastic response is determined by the equation

$$
P_{o}=\lambda_{o}^{-1}-\lambda_{o}^{-7}+\alpha\left(\lambda_{o}-\lambda_{o}^{-5}\right)=\phi\left(\lambda_{o}\right),
$$

say, where $\lambda_{o}=\lambda(0+)$ and $P_{o}=P(0+)$. Note that $\phi(x)=f(x, 0)$. As mentioned in Section 2 , the long term equilibrium response satisfies $P_{\infty}=\gamma \phi\left(\lambda_{\infty}\right)$.

The asymptotic values of $C(t)$ and $D(t)$ as $t \rightarrow \infty, \rightarrow \lambda(t) \rightarrow \lambda(\infty)$, can be found from (17a) and substituted into (18) to give $f(x, \infty)$. Depending on the values of $\alpha$ and $\lambda_{\infty}, f(x, \infty)$ may appear as in Figs. 2(b), 5(a) or 5(b). In view of (16) and (18), $\lambda_{\infty}$ also satisfies $P_{\infty}=F(x, \infty)$.

As a consequence of the redefinition of the original eqn (17)-(18), $f(x, \infty)$ and $\phi(x)$ may have different forms. It can be shown, however, that $f\left(\lambda_{\infty}, \infty\right)=\gamma \phi\left(\lambda_{\infty}\right)$.

\section{NUMERICAL RESULTS}

The numerical procedure for solving the governing equation (8) illustrates the analysis of Sections 4-8. The integrals in (17a) are approximated by expressions of the form

$$
\begin{aligned}
\int_{0}^{t} \dot{R}(t-s) f[\lambda(s)] \mathrm{d} s & =-\int_{t_{1}}^{t_{n}} \frac{\mathrm{d} R}{\mathrm{~d} s}\left(t_{n}-s\right) f[\lambda(s)] \mathrm{d} s \\
& \approx w_{n} f\left[\lambda\left(t_{n}\right)\right]+\sum_{k=1}^{n-1} w_{n k} f\left[\lambda\left(t_{k}\right)\right],
\end{aligned}
$$

where $\left(t_{1}=0, t_{2}, \ldots, t_{k}, \ldots, t_{n}=t\right)$ is some time partition. Weighting coefficients $w_{n}, w_{n k}$ depend on the values of the relaxation function $R\left(t_{n}-t_{k}\right)$ and the time increments, which are not necessarily equal. The integrals are approximated by Simpson's rule over intervals $\left[t_{k}, t_{k+2}\right]$. Three point forward, central and backward approximations are used for the derivative of $R\left(t_{n}-s\right)$ in this interval. For $n$ even, the trapezoidal rule was used on $\left[t_{1}, t_{2}\right]$ and the derivative was approximated by simple forward and backward difference expressions.

Substitution of the approximations (35) for $C$ and $D$ into (18) and rearranging gives

$$
\begin{aligned}
P\left(t_{n}\right) & =\frac{\sum_{n}^{C}}{x}-\frac{\sum_{n}^{D}}{x^{7}}+\bar{\alpha} x^{2}\left(\frac{1}{x}-\frac{1}{x^{7}}\right) \\
& =\hat{f}_{n}(x), \text { say, }
\end{aligned}
$$

where $\sum_{n}^{C}, \sum_{n}^{D}$ are the appropriate partial sums from (35) and $\bar{\alpha}=\alpha\left(1-w_{n}\right)$. Let $\hat{x}_{m}\left(t_{n}\right)$ satisfy $\mathrm{d} \hat{f}_{n}\left(\hat{x}_{m}\right) / \mathrm{d} x=0$. Starting with $\lambda\left(t_{1}\right)=1, P\left(t_{1}\right)=0,(36)$ was solved for $\lambda\left(t_{n}\right)$ with $1 \leq \lambda\left(t_{n}\right)<$ 
$\hat{x}_{m}\left(t_{n}\right)$, using a Newton-Raphson scheme. If for some $N$

$$
P\left(t_{N}\right)-\max \hat{f}_{N}(x)=P\left(t_{N}\right)-\hat{f}_{N}\left(\hat{x}_{m}\left(t_{N}\right)\right)>0,
$$

then another Newton-Raphson scheme was used to solve for time $t_{N^{*}}, t_{N-1}<t_{N^{*}}<t_{N}$ when the left hand side of (38) vanished. Clearly, $\lambda\left(t_{N^{*}}\right)=\hat{x}_{m}\left(t_{N^{*}}\right)$ and $\mathrm{d} \hat{f}_{N^{*}}\left(\hat{x}_{m}\left(t_{N^{*}}\right)\right) / \mathrm{d} x=0$. Thus, in view of the discussion at the end of Section $4, t_{N^{*}}$ is regarded as the bifurcation time. Equation (14) was used as a monitoring condition to anticipate impending bifurcation.

Computations were made with the Mooney parameter $\alpha=0.05$ and the relaxation function parameter $\gamma=0.25$. For this small value of $\alpha$ the response function was expected to have a well defined local maximum and minimum over a time interval long enough to ensure that the bifurcation condition would be met. Results for pressure histories $P(t)=0.5 t$ and $P(t)=3 t$ are shown in Fig. 6. The corresponding local maximum $f_{m}(t)=f\left(x_{m}(t), t\right)$ and stretch ratio histories are also shown. For each pressure history $f_{m}(t)$ decreases with $t$ as derived in result (vii). The intersection of the $P(t)$ and $f_{m}(t)$ graphs gives the bifurcation time. The difference between the values of $f_{m}(t)$ for the two pressure histories is too small to be shown on the graph. This is primarily due to the small value of $\alpha$. Figure 7 compares results for $P(t)=2 t, t \geq 0$, and when this history is changed to $P(t)=0.5, t \geq 0.25$. Even under continued expansion at constant pressure, the bifurcation condition is met. Of striking interest is the steepness of the stretch ratio histories near the bifurcation times. This arises from two sources. Firstly, the solution to (16) eventually lies in a domain near $x_{m}(t)$ where $f_{x}(x, t)$ is decreasingly rapidly. This is a region of rapid increase for $\lambda(t)$. Secondly, as $f(x, t)$ decreases with increasing $t$ the region of small slope broadens so that $\lambda(t)$ increases even faster.
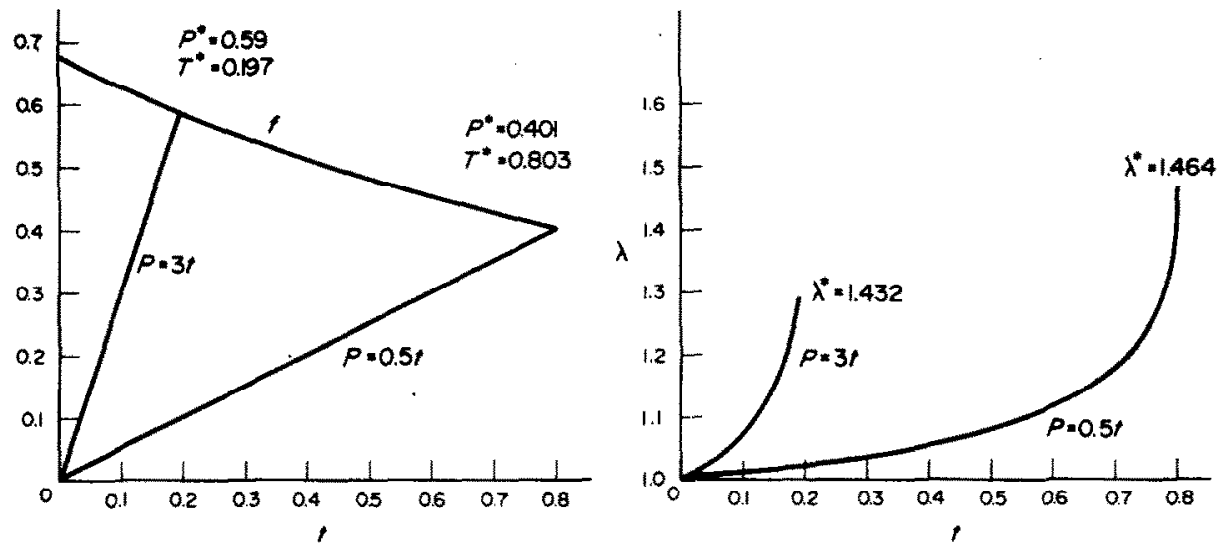

Fig. 6. Histories of $\lambda(t)$ and $f_{m}(t)$ for two different pressure histories up to the bifurcation time.
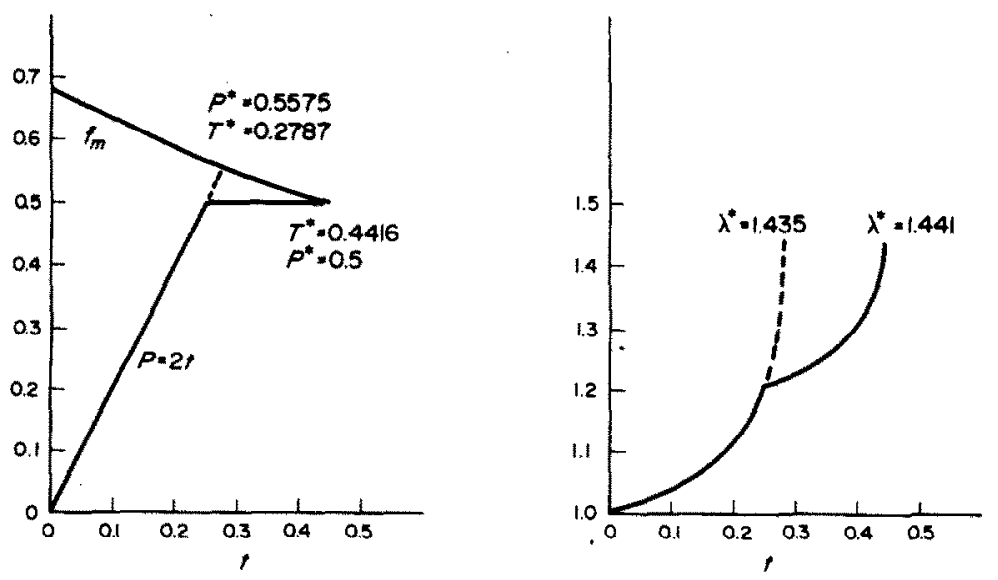

Fig. 7. Comparison of responses for a monotonic pressure history and one which is constant for $t>0.25$. 
In order to construct continuous extensions to the pre-bifurcation history $\lambda\left(t_{k}\right),(k=1,2$, $\ldots, N^{*}$ ), the pressure was reduced for $t_{n}>t_{N^{*}}$. Since $\sum_{N^{*}+1}^{C}$ and $\sum_{N^{*}+3}^{D}$ depend only on this pre-bifurcation history, $\hat{f}_{N^{*}+1}(x)$ is uniquely determined. For $P\left(t_{N^{*+1}}\right)<\max \hat{f}_{N^{*}+1}(x)$, (37) has two solutions, $\lambda^{-}\left(t_{N^{*}+1}\right)$ and $\lambda^{+}\left(t_{N^{*}+1}\right.$. Two past histories exist for $t_{n}>t_{N^{*}+2}$ producing two sets of coefficients $\sum_{n}^{C-}, \sum_{n}^{D-}, \sum_{n}^{C+}, \sum_{n}^{D_{+}}$and two response functions $\hat{f}_{n}^{-}(x)$ and $\hat{f}_{n}^{+}(x)$. For each $t_{n} \geq t_{N^{*}+2}, \lambda^{-}\left(t_{n}\right)$ and $\lambda^{+}\left(t_{n}\right)$ are the solutions of (36) using $\hat{f}_{n}^{-}(x)$ and $\hat{f}_{n}^{+}(x)$, respectively.

It was found that except for small $x, \hat{f}_{n}^{+}(x)<\hat{f}_{n}^{-}(x)$ and the condition function max $\hat{g}_{n}{ }^{+}(x)$ approached $\alpha$ faster than $\max \hat{g}_{n}{ }^{-}(x)$, as was discussed in section 6 . However, owing to the smallness of $\alpha$, the difference in the functions was small. In the negative slope region of (37), $\mathrm{d} \hat{f}_{n}^{+}(x) / \mathrm{d} x$ becomes very small and solution for $\lambda^{+}\left(t_{n}\right)$ becomes difficult. For this reason, $\lambda^{+}\left(t_{n}\right)$ was specified. Then $P\left(t_{n}\right)=\hat{f}_{n}^{+}\left(\lambda^{+}\left(t_{n}\right)\right)$ and $\lambda^{-}\left(t_{n}\right)$ was solved from (36) using $\hat{f}_{n}^{-}(x)$.

For some time interval beyond $t_{N^{*}}, \lambda^{+}\left(t_{n}\right)$ can increase. However, recalling Fig. 2(a), as $\max \hat{g}_{n}^{+}(x)$ approaches $\alpha$, the abscissae of the local maximum and minimum of $\hat{f}_{n}^{+}(x)$ approach each other. For this reason $\lambda^{+}\left(t_{n}\right)$ was decreased until the time $t_{n}$ when $\max \hat{g}_{n}{ }^{+}(x)<\alpha$. For larger times $\hat{f}_{n}^{-}(x)$ becomes monotonic and $\lambda^{+}\left(t_{n}\right)$ could be arbitrary. Since $\max \hat{f}_{n}^{-}(x)>$

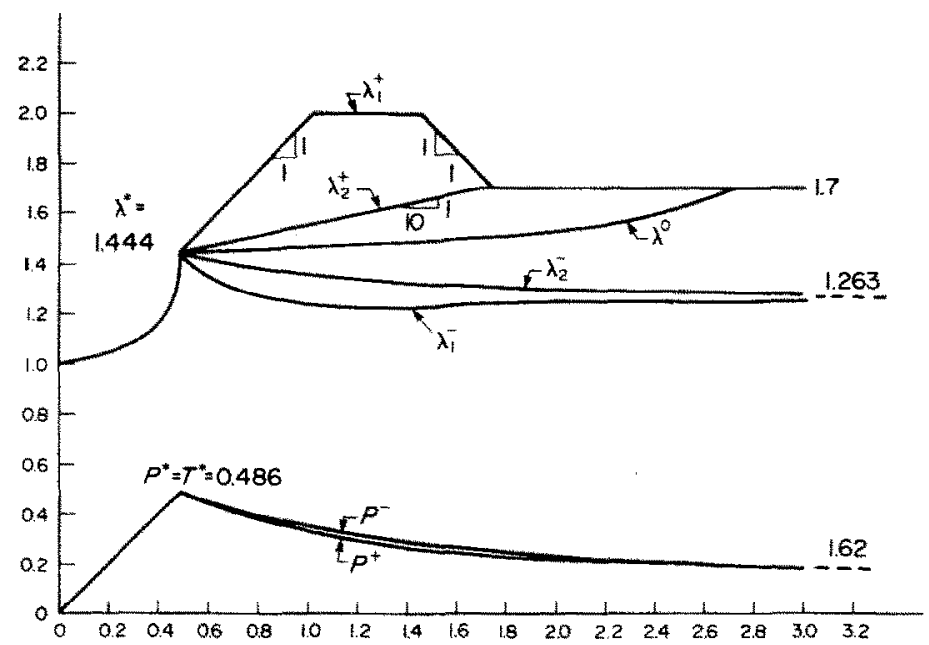

Fig. 8. Illustration of types of $\lambda^{+}(t)$ branch histories following bifurcation.

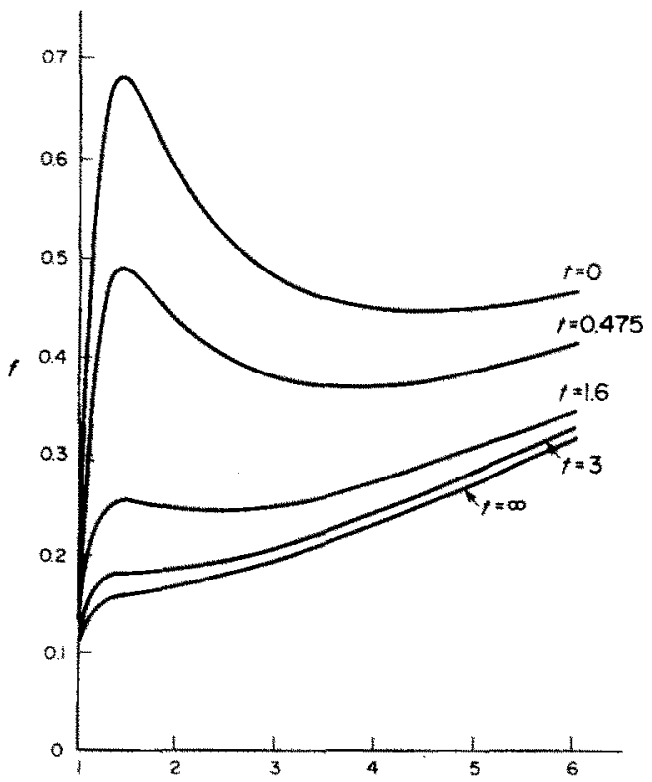

Fig. 9. Evolution of $f^{+}(x, t)$ corresponding to $\lambda_{1}^{+}(t)$. 
$\max \hat{f}_{n}^{+}(x), \lambda^{-}\left(t_{n}\right)$ lies in a region where $\mathrm{d} \hat{f}_{n}^{-}(x) / \mathrm{d} x$ is sufficiently large that an accurate solution to (36) could be found.

Figure 8 shows two sets of stretch ratio history branches following bifurcation due to pressure history $P(t)=t, t \leq T^{*}$. Branch $\lambda_{1}{ }^{+}(t)$ represents a branch which rapidly becomes large and must eventually be reduced. The monotonically increasing branch $\lambda_{2}{ }^{+}(t)$ is shown for comparison. $\lambda^{o}(t)$ is the solution of (30). It ceases to exist when $\max \hat{\mathrm{g}}_{n}{ }^{\circ}(x)=\bar{\alpha}$. The pressure histories corresponding to $\lambda_{1}{ }^{+}(t)$ and $\lambda_{2}{ }^{+}(t)$ differ only slightly, which shows the sensitivity of response following bifurcation.

Figure 9 shows the evolution of response functions $f^{+}(x, t)$ corresponding to $\lambda_{1}{ }^{+}(t)$ of Fig. 8 . In this case $g_{m}(\infty)<\alpha$, while $C^{+}(\infty)>0, D^{+}(\infty)>0$ so that $f^{+}(x, t)$ has the form shown in Fig. 2(b).

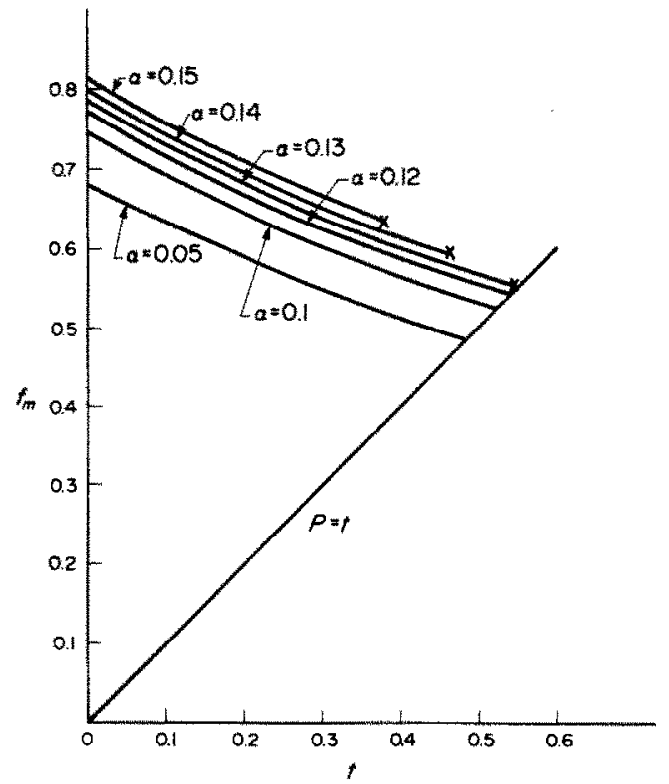

Fig. 10. The possibility for bifurcation vs values of $a$ for $P(t)=t$. The $X$ denotes when $f_{m}(t)$ ceased to exist.

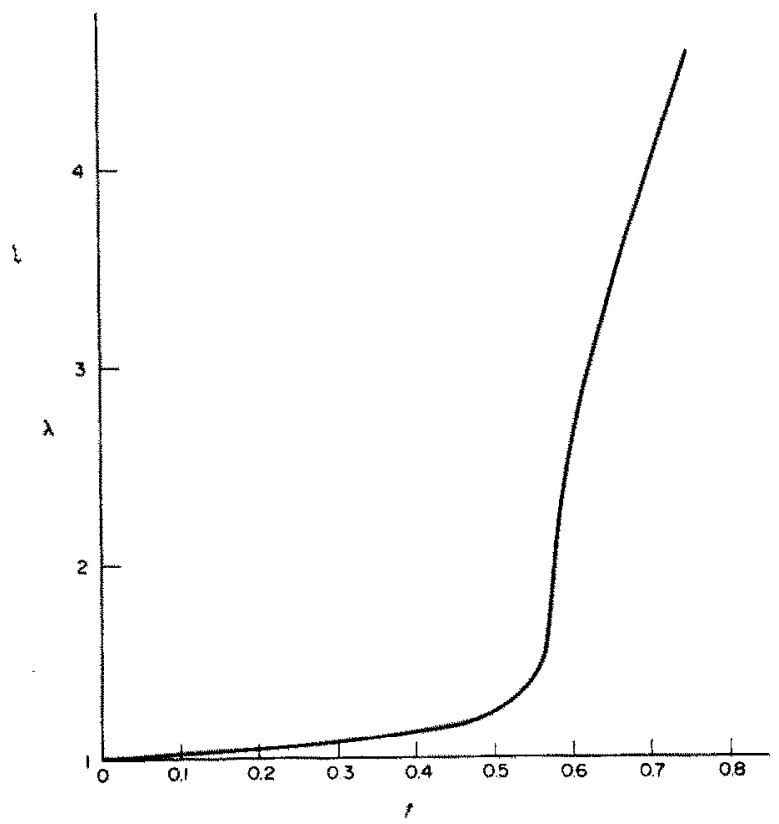

Fig. 11. Stretch ratio history for $P(t)=t, \alpha=0.15$, indicating rapid increase even though there was no bifurcation. 
Figure 10 shows $f_{m}(t)$ for various values of $\alpha$ when $P(t)=t$. When $f_{m}(t)$ intersects $P(t)$, response function $f(x, t)$ has a local maximum which exists long enough for (14a) to be satisfied and bifurcation to occur. When the $f_{m}(t)$ graph terminates in an $X, f(x, t)$ initially has a local maximum which ceases to exist at $t_{X}$ and (14a) can never be satisfied. The numerical results indicate that there is a critical value $\alpha^{*}$ of the Mooney parameter, slightly less than 0.13 , such that a bifurcation time exists if $\alpha<\alpha^{*}$ and does not exist if $\alpha<\alpha^{*}$.

These results suggest the conjecture that such a critical Mooney parameter exists for each monotonically increasing pressure history. Conversely, for each value of the Mooney parameter less than $\mathbf{0 . 2 1}$, there are pressure histories for which bifurcation is not possible.

Figure 11 shows the stretch ratio history when $P(t)=t$ and $\alpha=0.15$. Although $f(x, t)$ becomes monotonic before $P(t)$ becomes too large, it has a broad region of small slope. (Imagine $f\left(x, T^{*}\right)$ in Fig. 3 replaced by $f(x, t)$ in Fig. 2(b).) The steep portion of $\lambda(t)$ in Fig. 11 arises when the solution of (16) lies in this flat part of $f(x, t)$.

The steepness of the stretch ratio histories near their bifurcation times, in Figs. 6 and 7, and in Fig. 11 may be related to the apparent instability observed by Joye, Poehlein and Denson [6] in their experimental work on the inflation of flat membranes of certain polymers. This instability may in fact be a rapid change in shape corresponding to the steep parts of $\lambda(t)$ in Figs. 6,7 or 11, at least initially. If the expcrimental inflating pressure is not reduced, it may exceed some critical condition, analogous to the local maximum of a response function. Physically, this means that internal and external forces are no longer in balance and inertial effects arises. The membrane continues to inflate until the decrease in wall thickness leads to bursting. On the other hand, the experimental response may be better represented by the situation of Fig. 11. A critical pressure condition may not exist, but the increase in size becomes so large that failure again occurs.

Acknowledgement-The author is grateful for support for this work from a Rackham Research Fellowship of The University of Michigan and NSF Grant No. ENG 75-17489.

\section{REFERENCES}

1. A. E. Green and R. T. Shield, Finite elastic deformation of incompressible isotropic bodies. Proc. Roy. Soc. A202, 407 (1950).

2. A. E. Green and J. E. Adkins, Large elastic deformations and non-linear continuum mechanics. Oxford University Press, Oxford (1960)

3. A. S. Wineman, Large axially symmetric stretching of a nonlinear viscoelastic membrane. Int. J. Solids Structures 8, 775 (1972).

4. A. S. Wineman, Large axisymmetric deformation of a nonlinear viscoelastic membrane due to spinning. J. Appl. Mech. 39, 848 (1972).

5. L. E. Dickson, First Course in the Theory of Equations. Wiley, New York (1922).

6. D. D. Joye, G. W. Poehlein and C. D. Denson, A bubble inflation technique for the measurement of viscoelastic properties in equal biaxial extensional flow II. Trans. Soc. Rheo. 17, 287 (1973). 